\title{
Experimental investigations on implicatures: a window into the semantics/pragmatics interface
}

\author{
Napoleon Katsos \\ Research Centre for English and Applied Linguistics, University of Cambridge, UK \\ https://doi.org/10.36505/ExLing-2006/01/0034/000034
}

\begin{abstract}
It is traditionally assumed in the linguistic-pragmatic literature that Scalar Implica-tures (A or B $>>$ either A or B but not both; some of the Fs $>>$ at least one but not all of the Fs) are explicitly defeasible, structure-dependent and defeasible in context. We present three off-line studies that demonstrate the psycholinguistic reality of these properties of Scalar Implicatures (henceforth SIs). We then present two on-line text comprehension experiments that investigate the time-course of generating SIs and support a pragmatic account of SIs, according to which SIs are generated only when both structural and contextual constraints license them. We aim to demonstrate how an experimental approach can be informative on core issues in the seman-tics/pragmatics literature.
\end{abstract}

\section{Introduction}

Certain linguistic expressions form entailment scales where terms on the right of the scale are informationally stronger than terms on the left (measured by number of entailments, e.g. <some, most, all>, <or, and>; see Horn, 1984 i.a.). Scalar expressions may trigger Scalar Implicatures (SIs) when the assertion of an informationally weaker term conversationally implies (">>") the negation of the stronger terms in (1a \& b):

(1) a Mary: Who is representing the company at the court hearing? John: Turner or Morris. > Either Turner or Morris but not both.

b Mary: How is our candidate doing in the polls?

John: He has managed to overtake some of his opponents that have little funding. > At least one but not all them.

Characteristic properties of SIs include explicit defeasibility, structuredependency and defeasibility in context (Gazdar, 1979; Horn, 1984 i.a.). The fact that SIs share some properties of grammatical inferences has given a rise to a debate on how to classify them: as structure-based default inferences (Chierchia 2004; Levinson 2000 i.a.) or truly context-dependent pragmatic inferences (Atlas, 2005; Carston, 2002; Grice, 1975; Hirschberg 1991;

ExLing 2006: Proceedings of 1st Tutorial and Research Workshop on Experimental Linguistics, 28-30 August 2006, Athens, Greece 
Recanati, 2003; Sauerland, 2004; Sperber \& Wilson, 1995 i.a.). In the first part of the paper we present three off-line studies that demonstrate the psycholinguistic reality of the properties of SIs. In the second part of the paper we present two on-line studies that address the debate between default and pragmatic theories.

\section{On the properties of SIs Explicit defeasibility}

The first off-line study investigates whether SIs are defeasible inferences, i.e. whether they can be explicitly revised without giving rise to contradictions. The baseline condition for defeasibility is the entailment, a grammatical inference whose contradiction ought to give rise to a strong contradiction.

Participants were asked to rate short question/answer pairs for coherence. The critical items consisted of a question and an answer that came in two utterances. The first utterance of the answer contained a disjunction in upward-entailing structure that licenses the generation of an SI. The second utterance of the answer revised an aspect of the meaning of the disjunction. In the Implicature condition, the second utterance contradicted the content of the SI of the disjunction (2a). In the Entailment condition, the second utterance contradicted the content of the entailment of the disjunction (2b).

(2) a The director asked his consultant: Who is representing our company at the court hearing? His consultant replied: Turner or Morris. In fact, both of them are.

b The director asked his consultant: Who is representing our company at the court hearing? His consultant replied: Turner or Morris. In fact, none of them are.

Analyses of variance indicate that revising the implicature is significantly more acceptable than revising an entailment (5.7 vs 1.3 on a 7 point scalar where 7 indicates that the answer is 'perfectly coherent'; $F 1(1,24)=$ $445.3, p<.001 ; F 2(1,16)=990.2, p<.001)$. This is evidence that implicatures are explicitly defeasible in a way that truly grammatical inferences are not.

\section{Structure-dependency and defeasibility in context}

It is also argued in the linguistic literature that SIs are constrained by structural and contextual factors. SIs are generated in conditions where both structure and context license them, i.e. in Upward-entailing structures with 
Upper-bound contexts; in this condition the disjunction should be interpreted exclusively, with an SI (3a). However, SIs are not available in conditions where contextual constraints do not license them, i.e. in Upward-entailing structures with Lower-bound contexts, where the Lower-bound context biases towards an inclusive interpretation of the disjunction without an SI (3b). Furthermore, SIs are not generated when linguistic structure doesn't license them, i.e. in Downward-entailing structures (e.g. in the antecedent of a conditional, as in 3c):

(3) a UB: The director asked his consultant: Who is representing our company at the court hearing? His consultant replied: Turner or Morris from the Legal Department.

b LB: The director asked his consultant: Who is available to represent our company at the court hearing? His consultant replied: Turner or Morris from the Legal Department.

c DE: The director asked his consultant: Who is representing our company at the court hearing? His consultant replied: I believe that if Turner or Morris from the Legal Department do so, we need not worry too much.

In the second off-line study, participants were asked to rate on a scale whether they believe that the answer implies ' $\mathrm{X}$ or Y but not both of them', or whether they believe that the answer implies ' $\mathrm{X}$ or $\mathrm{Y}$ and even both of them'. In the third off-line study, participants were asked to fill in a verb inflected for number at the end of the last utterance. We assumed that if they interpreted the disjunction with an SI, they would use a verb form inflected in singular (e.g. X or Y is), whereas if the interpreted the scalar term without an SI, they would use a verb inflected in plural (e.g. X or Y are).

With regards to the second study, the disjunction was judged as exclusive in UB and inclusive in LB and DE (2.9 vs 5.2 and 5.1 respectively in a 7 point scale, where 1 indicates that the disjunction was exclusive). Analyses of variance indicate a main effect of condition $F 1(2,26)=37.5, p<.001 ; F 2(2,14)=$ $23.7, p<.001$. Planned comparisons reveal that UB is judged significantly more exclusive and that $\mathrm{LB}$ and $\mathrm{DE}$ are equally inclusive ${ }^{1}$.

With regards to the third study, participants used a verb in singular agreement $82.1 \%$ in UB, $49.1 \%$ in LB and $47.9 \%$ in DE. There was a main effect of Condition $(F 1(2,38)=77.3, p<0.001 ; F 2(2,14)=48.4, p<$ $0.001)$. Planned comparisons show that there was a significant difference between the UB and the LB conditions and the UB and DE conditions whereas the difference between the LB and DE conditions was not significant ${ }^{1}$. We conclude that SIs are generated in UB, where the inference is li- 
censed both by context and structure, but not generated in LB and DE conditions where either the context or the structure don't license the SI.

\section{The debate between default and pragmatic accounts}

The off-line studies show that SIs are indeed explicitly defeasible, structuredependent and defeasible in context. In the final part of the paper we present two on-line studies that address the debate on the default vs pragmatic nature of SIs. Default accounts (Chierchia 2004; Levinson 2000 i.a.) claim that SIs are generated by default when licensed by structural constraints (in UB and LB), and may have to be cancelled in subsequent stages if not licensed by the context (in LB). Pragmatic accounts claim that SIs are generated only when both structure and context license them. In case the context doesn't license the SI, the SI is simply not generated, rather than generated and then cancelled. Two studies investigated the on-line processing of disjunctions (with items similar to $3 \mathrm{a} \& \mathrm{~b}$ ) and the existential quantifier.

Reading time results for the disjunction indicate that processing the scalar term with an SI in the UB condition is more time consuming than processing the scalar term without an SI in the LB condition $(811 \mathrm{~ms}$ vs $761 \mathrm{~ms} ; F 1$ $(1,36)=6.053, p<0.02 ; F 2(1,15)=8.612, p=0.01)$. Similar results were obtained for the existential quantifier ${ }^{1}$. It is impossible to argue that in the LB condition the SI was generated by default and then cancelled, in less time than the SI was generated in the UB condition. On the other hand, these findings are consistent with pragmatic accounts that predict that SIs are generated only when licensed by both structure and context in UB, whereas they are simply not generated at all when they are not licensed by context in LB.

\section{Conclusion}

We presented three studies that demonstrate the psycholinguistic reality of traditional linguistic-pragmatic intuitions on SIs. We also presented two online studies that are informative on the recent debate on the default vs contextual nature of SIs. We discuss these findings with regards to literature in sentence processing and the acquisition of semantic/pragmatic competence, and we illustrate how the experimental approach can contribute to issues in the core of linguistic theory.

\section{Notes}

Please contact the author for appendices with the items, statistical analyses of the planned comparisons and a list of references. 